\title{
New product launch: herd seeking or herd preventing?
}

\author{
Ting Liu • Pasquale Schiraldi
}

Received: 26 November 2009 / Accepted: 24 February 2011 / Published online: 26 March 2011

(C) Springer-Verlag 2011

\begin{abstract}
A decision maker offers a new product to a number of potential adopters. He does not know the value of the product, but adopters receive some private information about it. We study how the decision maker may influence learning among adopters by manipulating the launch sequence when both the decision maker and adopters can learn about the value of the product from previous adoption decisions. The conditions under which the decision maker prefers a sequential launch to a simultaneous launch depend on adopters' prior beliefs about the value of the product and adoption costs. We derive the decision maker's optimal launch sequence and study how it endogenizes informational herding.
\end{abstract}

Keywords Herding $\cdot$ Product launch sequence $\cdot$ Social learning

\section{JEL Classification D8}

\begin{abstract}
An earlier version of this paper was circulated under the title "Monopoly's New Product Launch Strategy: herd seeking or herd preventing?" We are very grateful to Ching-to Albert Ma, Jacob Glazer, Barton Lipman, Christophe Chamley, Thomas Jeitschko, Heski Bar-Isaac, Daniel Sgroi and participants at the Microeconomics Theory Workshop at Boston University. We also thank Johannes Hörner and an anonymous referee for their valuable suggestions.
\end{abstract}

T. Liu

Department of Economics, Michigan State University,

East Lansing, MI 48824, USA

e-mail: tingliu@msu.edu

P. Schiraldi $(\varangle)$

Department of Economics, London School of Economics,

Houghton St., London WC2A2AE, UK

e-mail: p.schiraldi@1se.ac.uk 


\section{Introduction}

Anecdotal evidence shows that people's decisions are often influenced by others' choices. Moviegoers are easily attracted to movies with big opening box offices; a farmer's decision to adopt a new technology is affected by the number of early adoptions; in presidential elections, a "momentum" effect is well documented. The fact that later adopters may herd on early adopters' choices posts a natural question to a decision maker who wants to maximize the total number of adoptions. Should he ask adopters to decide simultaneously or sequentially? If a sequential launch is preferred, what is the optimal launch sequence? The decision maker could be a firm introducing a new product to many markets, a government promoting a new agriculture technology among farmers, or a health care administrator promoting a new procedure among doctors.

This paper studies how a decision maker should optimally introduce a new product to potential adopters in the presence of herd behavior, resulting from social learning. Adopters have a common value but each one receives private information regarding the unknown common value. Early adopters' decisions are based on their private information. As a result, subsequent adopters may infer early adopters' private information from their choices. When public information swamps an adopter's private information, all the remaining adopters herd on the same action.

Initiated by Banerjee (1992) and Bikhchandani et al. (1992), most existing social learning literature studies how a herd naturally arises when adopters make decisions sequentially in an exogenous order. We argue that a decision maker can influence learning among adopters and hence the formation of a herd by manipulating the launch sequence. For example, when launching a new movie, a movie studio can choose between a national release or a sequential release; a government promoting a new technology among farmers can ask the farmers to make the adoption decisions at the same time or sequentially. Furthermore, the decision maker can control the amount of information revealed in a sequential launch. For instance, very little information about the product will be revealed if only one adopter is asked to make a decision before all others. By contrast, if the decision maker asks a hundred adopters to make decisions in the first period, a significant amount of information about the product will be revealed to the remaining adopters.

In our model, the value of the product is unknown to adopters and the decision maker. This is because the value of the product represents the match between adopters' tastes and the underlying characteristics of the product. Each adopter receives a signal regarding the common value. The signal remains an adopter's private information while his adoption decision becomes public information. The decision maker decides how many offers to make in each period contingent on the history.

The optimal launch strategy depends on the prior belief that the product is of high value and the adoption cost faced by potential adopters. We say that the product is promising when the prior belief exceeds one-half but is not too close to unity; it is unpromising when the prior belief is smaller than one-half but is not too close to zero. The decision maker's optimal strategy is summarized as follows: first, the decision 
maker is indifferent between a sequential launch and a simultaneous launch when the prior belief is either extremely high or extremely low. Second, the decision maker prefers a sequential launch to a simultaneous launch when the product is promising or when it is unpromising but cheap to adopt. Finally, the decision maker prefers a simultaneous launch to a sequential launch when the product is unpromising and costly to adopt.

When the prior belief is extreme, the decision maker cannot increase the number of adoptions by manipulating the launch sequence. This is because in a sequential launch, early adopters' private information is dominated by the extreme prior belief, and hence, their decisions are always in accordance with the prior belief. Now that later adopters do not learn any additional information in a sequential launch, their decisions will be the same as in a simultaneous launch.

By contrast, launch strategies will make a difference when the prior belief is not extreme. In this case, early adopters' decisions are based on their private information. The additional information conveyed by early adopters' decisions may change later adopters' minds. As a result, later adopters' decisions in a sequential launch may differ from those in a simultaneous launch. To convince the remaining adopters that the product is worth taking, the decision maker needs to ensure that enough adopters have adopted the product, but this depends on whether the product is promising, that is, whether the prior belief that the product has a high value is greater than one-half.

When the product is promising, the decision maker will always launch it sequentially despite a large demand in a simultaneous launch. This is because early launches are more likely to deliver convincing good news and hence induce later adopters with bad signals to adopt the product as well. Essentially, when launching a promising product, the decision maker wants adopters to learn from each other and make more informed decisions. By the law of large numbers, the more offers the decision maker makes today in a sequential launch, the higher the chance that an adoption herd will occur tomorrow. However, since fewer adopters are left, the benefit from an adoption herd is smaller. The optimal number of offers made in a given period depends on the trade-off between a larger chance of an adoption herd tomorrow and a smaller benefit from an adoption herd.

When launching an unpromising product, the decision maker will offer it simultaneously to all adopters if the adoption cost is high; he will offer it to one adopter first and then to all the remaining adopters if the adoption cost is low. In other words, the decision maker either does not allow adopters to learn from each other's decisions at all or allows them to learn the minimum amount of information. This is because early launches are more likely to deliver bad news and trigger a rejection herd. Therefore, the decision maker does not want to reveal too much information. It is worth noticing that a rejection herd will never arise when the product is unpromising due to the decision maker's cautious launch strategy. This is in stark contrast with the prediction of the herding literature with an exogenous launch sequence. There, a rejection herd occurs with a positive probability.

Our model sheds some light on a firm's new product launch strategy when the price of the product is fixed. It fits situations where the price is regulated, or it is too costly to adjust price frequently. Examples include the pharmaceutical industry and 
the motion picture industry. ${ }^{1}$ In the motion picture industry, blockbusters are often released nationally while good artistic movies are often released first in a few big cities and then rolled out to other cities. The movie Borat (2006) was released in a limited fashion and soon topped the box office. Bruce Snyder, Twentieth Century Fox President of Distribution, commented on the limited release of Borat: "Not only is there an existing audience that cannot wait to see the film, what we have here is a movie with an incredible amount of playability. Through a tiered release pattern, we'll be able to build a huge amount of momentum."2 Our prediction is consistent with Bruce Snyder's comment and provides one explanation for why many well-anticipated products are released sequentially even when a sequential launch involves a possible waiting cost and involves the risk of losing customers to rivals.

One interesting implication of the model is that a promising product may have a higher failure rate than an unpromising product. When launching a promising product, the decision maker is confident enough to reveal a lot of information in an attempt to induce an adoption herd. However, if it turns out that very few people adopt the product at the early stage, the decision maker will be trapped in a rejection herd. By contrast, when launching an unpromising product, the decision maker will never reveal much information for fear of a rejection herd. Hence, remaining adopters will never hear bad news that is damaging enough to kill the product.

Our model also generates the following implications. First, a promising product has a longer launch duration than an unpromising product. Second, the decision maker will reveal more information during the launch of a promising product than during the launch of an unpromising product. Third, when adopters' signals are more precise, the decision maker is more likely to launch the product sequentially to influence later adopters' decisions. And finally, if the signals drawn by adopters have different precision levels, the decision maker will offer the product first to those with more precise signals.

The paper is organized as follows. Section 2 discusses the related literature. Section 3 introduces the model. Section 4 illustrates the basic idea with a simple model. Section 5 shows the main results and discusses related issues. Section 6 concludes. All proofs are gathered in the Appendix.

\section{Related literature}

Bikhchandani et al. (1992), and Banerjee (1992) are the pioneer works in the social learning literature. They show that herds can be rational but rational herds may contain very little information. In their models, adopters make decisions in an exogenous sequence. By contrast, we allow the decision maker to determine the launch sequence.

Bose et al. $(2008,2006)$ extend the literature of social learning by allowing a monopolist to affect the information aggregation over time by changing the price. In their model, the monopolist faces the trade-off between current rent extraction and

\footnotetext{
${ }^{1}$ Movie tickets are priced almost the same across all regions within the United States.

2 "Borat Tiered Theatrical Release Maximizes Playability With Core Audience", Movie City News, Oct. 23, 2006.
} 
future information screening. We instead endogenize the sequence in which consumers arrive. The seller's trade-off in our model is between the public information generated by earlier adoptors and the profit gain (loss) from those earlier adopters due to their less-informed decisions.

This paper is related to Sgroi (2002) who studies how a firm can influence the herd by choosing the number of consumers to serve in the first period. Our paper differs from his in two ways. Sgroi studies a case where the seller knows the quality of the product while consumers learn it over time. By contrast, in our model, both the decision maker and adopters learn the value of the product over time. Second, in Sgroi's, the firm can only affect the herd in the first period so that the model constitutes a static optimization problem. We allow the decision maker to influence the herd in each period, contingent on early adopters' decisions. To the best of our knowledge, this is the first paper to study how a decision maker can influence the herd through the sequencing decision in a dynamic setting. The dynamic structure allows us to generate the decision maker's equilibrium path, thus, providing richer predictions.

In a very recent paper, Bhalla (2008) extends our model and studies how a monopolist should introduce a product by choosing both price and sequencing. With a flexible price, Bhalla reports a launch pattern similar to ours. Nevertheless, there are three differences between her results and ours. First, we find the decision maker should launch the product simultaneously when the prior belief is low but not extremely low while Bhalla shows the firm should release the product sequentially. This is because with a fixed price, the decision maker bears a high risk of a rejection herd by launching an unpromising product sequentially. By contrast, in Bhalla, the firm does not bear the risk because in case of a failed early launch, it can reduce the price to reflect the updated value and sell the product again. Second, our model predicts that a promising product's launch pattern is drastically different from an unpromising product whereas Bhalla does not differentiate the monopolist's launch strategy based on whether the product is promising or not. Third, due to the decision maker's strategy, in our model, a rejection herd may occur with a promising product but will never occur with an unpromising product. So, a promising product may have a higher chance of a complete failure than an unpromising product. In Bhalla, regardless of whether the product is promising or not, the firm can always prevent a rejection herd by reducing the price.

Bar-Isaac (2003) studies a dynamic learning model where a seller is privately informed about the quality of the product whereas buyers learn the quality of the product over time. By contrast, in our paper, buyers are privately informed about the quality of the product and both the seller and buyers learn the quality of the product over time. In our model, buyers' willingness to pay for the low-quality product is zero. Hence, if the seller is informed about the quality of the product, the low-quality seller will always mimic the launch strategy of the high-quality seller. So, both types of sellers will always launch the product sequentially. This is different from Bar-Isaac's result that a good seller will always sell the product but a bad seller may choose not to sell the product when reputation falls below some threshold. The driving force behind the difference is that we assume zero production cost whereas Bar-Isaac assumes a positive production cost. Another departure from Bar-Isaac is that the seller in our model not only decides whether to trade in each period but also decides how many offers to make in each period. 
Other articles that model how a monopolist maximizes profit by manipulating the information available to consumers include Ottaviani and Prat (2001), Gill and Sgroi (2008a,b), Taylor (1999), and Bergemann and Valimaki (1996, 1997, 2000). We extend this strand of literature by studying how a decision maker influences learning among adopters by choosing the launch sequence optimally.

Bala and Goyal (2001) and Coopland (2007) study how the survival and adoption of technologies depend on heterogeneous beliefs and social learning (Coopland 2007) as well as local interaction between agents (Bala and Goyal 2001). However, they completely abstract from any strategic decision by the decision maker.

\section{The model}

A risk-neutral decision maker offers a new product to $N$ potential adopters and wishes to maximize the total number of adoptions. The adopters are indexed by $i \in\{1,2, \ldots, N\}$. The decision maker considers offering the product to these adopters over time, which we take as discrete. The index $t \in\{1,2, \ldots\}$ denotes the period in which the decision maker may offer the product. The decision maker will offer the product to an adopter at most once. If the product is offered to adopter $i$, the adopter then decides whether to take the product.

An adopter's cost of adoption is fixed at $c$, which is commonly known. ${ }^{3}$ Let $n_{t}$, with $n_{t} \in\{0,1,2, \ldots, N\}$, denote the number of offers the decision maker makes in period $t$. The decision maker chooses $n_{t}$ in period $t$. For example, he may offer the product to adopters 1 to 3 in period 1 and then to adopters 4 to $N$ in period 2. Alternatively, the decision maker may offer the product to one adopter in each period.

The value of the product $v$ is either low or high, $v \in\{L, H\}$. Without loss of generality, we let $L=0$ and $H=1$. The value $v$ is unknown to the decision maker and adopters. The value may represent the match between the underlying characteristics of the product and adopters' tastes. The decision maker does not know adopters' tastes, and adopters do not know the underlying characteristics of the product. In period $t$, the public belief that the product has a high value is $\lambda_{t}$, with $\lambda_{t} \in(0,1)$. An adopter adopts at most one unit of the product. His utility is $v-c$ if he takes the product and 0 otherwise.

When the product is offered to adopter $i$, a random signal $s_{i}$ is generated and received by this adopter. Signal $s_{i}$ remains adopter $i$ 's private information. Conditional on the true value $v$, signals received by different adopters are independent and identically distributed. Let $s_{i} \in\{L, H\}$ be the possible values of the signal, where $s_{i}=L$ indicates a "bad" signal while $s_{i}=H$ indicates a "good" signal, respectively:

$$
\operatorname{Prob}\left(s_{i}=H \mid v=1\right)=\operatorname{Prob}\left(s_{i}=L \mid v=0\right)=q, \forall i,
$$

\footnotetext{
3 In the context of new technology adoption, the cost is the value of the existing technology. In the context of a firm selling a new product, the cost represents the fixed price of the product.
} 
where $q \in(0.5,1)$. That is, when adopters receive signal $H$, the value of the product is more likely to be high than when they receive signal $L$. The parameter $q$ is common knowledge and is called the precision of a signal. Note that $q$ does not depend on adopters' identities. We will discuss what happens when adopters' signals vary in precisions in Sect. 5.

The sequence of events in period $t$ is as follows. First, the decision maker and the remaining adopters observe the decision maker's previous decisions including how many offers are made and the number of adoptions in each previous period. Second, the decision maker decides $n_{t}$, the number of offers to make in period $t$. Third, if the decision maker offers the product to adopter $i$, this adopter receives a private signal, $s_{i}$, about the value of the product and he decides whether to take the product.

An adopter will make a decision at most once, and therefore, his decision rule is very simple. When he is offered the opportunity to adopt the product, his decision is a function of the public belief in that period and his private signal. The adopter will adopt the product if and only if his posterior belief is greater than or equal to the cost of adoption.

The decision maker can observe everything that happened in previous periods, but in equilibrium, his strategy bases only on the public belief and the number of the remaining adopters in the current period. Let $n^{*}\left(\lambda_{t}, N_{t}\right)$ denotes the decision maker's equilibrium action in period $t$, and let $V\left(\lambda_{t}, N_{t}\right)$ denote the decision maker's value function. Given adopters' strategies, $n^{*}\left(\lambda_{t}, N_{t}\right)$ maximizes the expected number of adoptions.

We make the following tie-breaking rules. First, when the decision maker is indifferent between offering the product to an adopter now or in the future, he offers it now. Second, the decision maker will not offer the product to an adopter if he will reject the product. The former assumption can be justified by a small waiting cost for the decision maker, and the latter assumption can be justified by a small cost of making the offer.

We define some notation here. Given a prior belief $\lambda$, adopter $j$ 's posterior belief after receiving a bad signal is

$$
\lambda^{L}(\lambda) \equiv \operatorname{Pr}\left(v=1 \mid \lambda, s_{j}=L\right)=\frac{\lambda(1-q)}{\lambda(1-q)+(1-\lambda) q} .
$$

Similarly, the adopter's posterior belief upon receiving a good signal is

$$
\lambda^{H}(\lambda) \equiv \operatorname{Pr}\left(v=1 \mid \lambda, s_{j}=H\right)=\frac{\lambda q}{\lambda q+(1-\lambda)(1-q)} .
$$

Figure 1 characterizes an adopter's posterior beliefs as functions of his prior belief. The concave curve represents $\lambda^{H}(\lambda)$, and the convex curve represents $\lambda^{L}(\lambda)$. Because the value of the product is $v \in\{0,1\}$, the adopter's expected value of the product after receiving a good signal is $\lambda^{H}(\lambda)$ and his expected value of the product after receiving a bad signal is $\lambda^{L}(\lambda)$. 


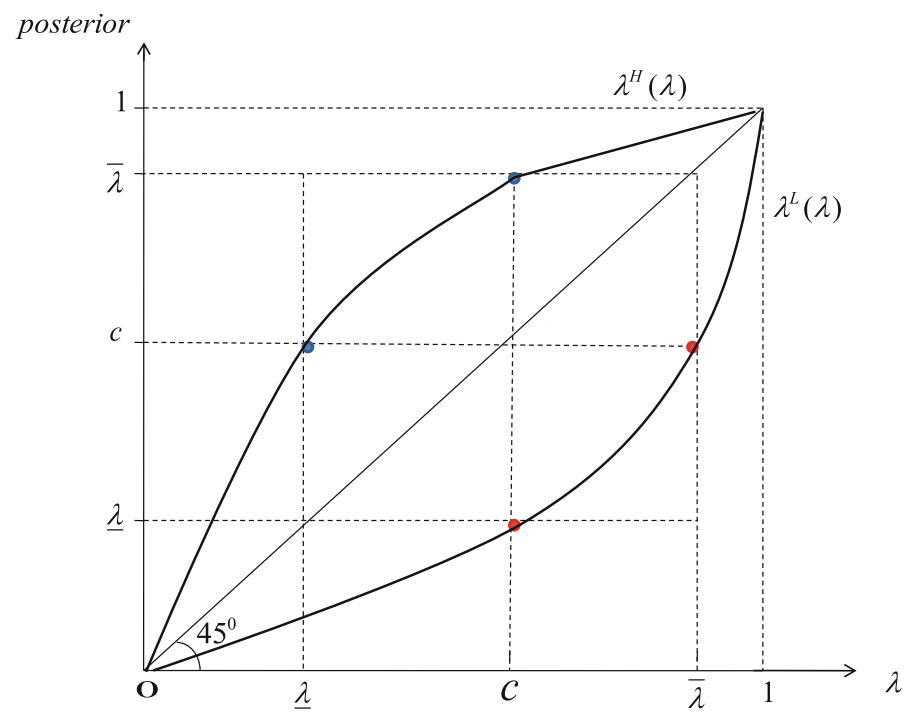

Fig. 1 Posterior beliefs

\section{An example with two adopters}

In this section, we use a simple example with two adopters to illustrate when the decision maker prefers a sequential launch to a simultaneous launch, and if so, how much he gains by a sequential launch. The decision maker's launch strategy critically depends on adopters' prior beliefs and adoption costs.

In the example, we drop the subscript $t$. Let $\lambda$ denotes the prior belief. The discussion is divided into four cases according to the level of the prior belief (see Fig. 2). The cases are ordered by extremely pessimistic adopters $(\lambda<\underline{\lambda})$, pessimistic adopters $(\underline{\lambda}<\lambda<c)$, optimistic adopters $(c<\lambda<\bar{\lambda})$, and extremely optimistic adopters $(\overline{\bar{\lambda}}<\lambda)$. The decision maker will launch the product sequentially, i.e., encourage learning among adopters, only when adopters are optimistic about the product.

To begin, we define two threshold beliefs

$$
\begin{aligned}
& \bar{\lambda} \equiv \frac{c q}{c q+(1-c)(1-q)}, \\
& \underline{\lambda} \equiv \frac{c(1-q)}{c(1-q)+(1-c) q} .
\end{aligned}
$$

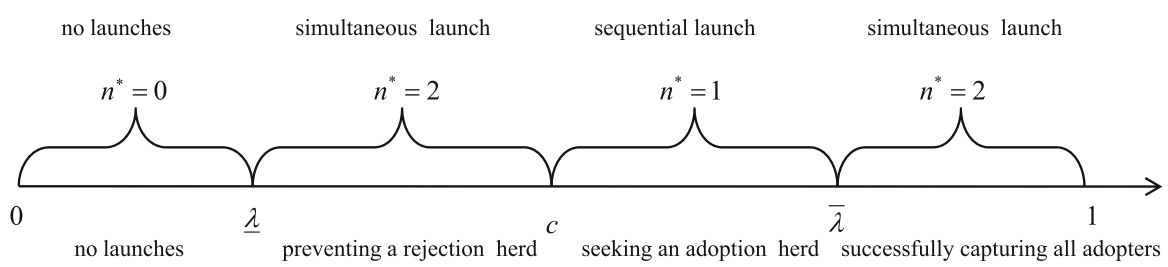

Fig. 2 An example with two adopters 
At belief $\underline{\lambda}$, an adopter is indifferent between taking and rejecting the product upon receiving a good signal; ${ }^{4}$ at belief $\bar{\lambda}$, an adopter is indifferent between taking and rejecting the product upon receiving a bad signal. ${ }^{5}$

No launches $(\lambda<\underline{\lambda})$ : When adopters are extremely pessimistic, the decision maker will never launch the product. At the prior belief $\underline{\lambda}$, an adopter is indifferent between taking and rejecting the product upon receiving a good signal, i.e., $\lambda^{H}(\underline{\lambda})=c$. Since the posterior belief $\lambda^{H}(\lambda)$ increases in the prior belief, when an adopter's prior belief is below $\underline{\lambda}$, he will not take the product even if he receives a good signal, i.e., $\lambda^{H}(\lambda)<c$ (see Fig. 1). Hence, the decision maker's payoff from a simultaneous launch is zero.

In a sequential launch, the first adopter will always reject the product. The second adopter therefore cannot infer any information from the first adopter's decision and will make the same decision as his predecessor. So, the decision maker's payoff from the sequential launch is also zero. Given the tie-breaking rule, the decision maker will not launch the product.

Preventing a rejection herd $(\underline{\lambda}<\lambda<c)$ : Pessimistic adopters think that the product is not worth adopting before receiving any additional information. When the product is offered to an adopter, he will take it if and only if his signal is good, i.e., $\lambda^{L}(\lambda)<c<\lambda^{H}(\lambda)$ (see Fig. 1). The decision maker prefers to launch the product simultaneously to prevent a rejection herd. In fact, the information conveyed by the first adopter in a sequential launch could only hurt the decision maker.

In a sequential launch, at best, the second adopter will observe an adoption by the first adopter and hence infer that he receives a good signal. A good signal, however, is not strong enough to convince the second adopter to adopt the product if his own signal is bad. Because adopters' signals are equally precise, opposite signals cancel out each other ${ }^{6}$, and hence, the posterior belief remains the same as the prior belief. By contrast, an unsuccessful launch in the first period will trigger a rejection herd. If the first adopter rejects the product, the second adopter will reject it as well even if his own signal is good.

In particular, the decision maker loses $q(1-q)$ from a sequential launch, where $q(1-q)$ is the probability that the first adopter receives a bad signal and the second adopter receives a good signal. Notice, the loss from a sequential launch decreases in the precision of the signal. As the precision of the signal increases, it is unlikely for the two adopters to receive conflicting signals; therefore, the chance of a rejection herd in a sequential launch becomes smaller.

Seeking an adoption herd $(c<\lambda<\bar{\lambda})$ : If adopters are optimistic, they think the product is worth adopting before receiving any additional information. Again, an adopter will take it if and only if his signal is good (see Fig. 1). In this case, the decision maker will launch the product sequentially to seek an adoption herd.

\footnotetext{
$4 \underline{\lambda}$ is derived by setting expression (2) to $c$.

$5 \bar{\lambda}$ is derived by setting expression (1) to $c$.

${ }^{6} \lambda^{L}(\lambda)=\frac{\lambda(1-q)}{\lambda(1-q)+(1-\lambda) q}, \lambda^{H}\left(\lambda^{L}(\lambda)\right)=\frac{\lambda^{L}(\lambda) q}{\lambda^{L}(\lambda) q+\left(1-\lambda^{L}(\lambda)\right)(1-q)}=\lambda$.
} 
The analysis is similar to the case of pessimistic adopters. A sequential launch differs from a simultaneous launch only when the first adopter receives a good signal while the second adopter receives a bad signal. In this case, the good news conveyed by the predecessor's adoption will change the second adopter's mind and convince him to adopt the product as well —a little more good information will trigger an adoption herd. By contrast, a rejection by the first adopter is not bad enough to trigger a rejection herd. If his private information is favorable, the second adopter will still take the product.

Successfully Capturing All Adopters $(\bar{\lambda}<\lambda)$ : When adopters are extremely optimistic, they will take the product regardless of their private signals. The argument is similar to the case of extremely pessimistic adopters. Given the tie-breaking rule, the decision maker will launch the product simultaneously.

While the two-adopter example illustrates the trade-off between sequential and simultaneous launches, new issues arise with more than two adopters. With three or more adopters, the decision maker's strategy is richer. He may find it optimal to offer the product to more than one but fewer than all adopters, choosing an interior solution to control the information revealed to the remaining adopters.

\section{The equilibrium}

Now, we analyze the decision maker's equilibrium strategy in the general case when there are $N$ adopters, where $N>2$. When there are just two adopters, the decision maker can only decide whether to release information. The general case allows us to study how much information the decision maker would like to reveal during a sequential launch.

We call a product unpromising if $\underline{\lambda}<\lambda_{t}<\frac{1}{2}$ and promising if $\frac{1}{2}<\lambda_{t}<\bar{\lambda}$. Hence, the decision marker's strategy is based on the following intuition: the adopters being optimistic or pessimistic determines whether one good (bad) signal is sufficient to trigger an adoption (rejection) herd. Differently, whether a product is promising determines whether allowing learning, i.e., offering the product to many adopters in a single period, is more likely to convey good news or bad news about the quality of the product to future adopters.

The decision maker's equilibrium action in period $t$ depends on the public belief $\lambda_{t}$ and the number of remaining adopters $N_{t}$. We denote the decision maker's equilibrium action in period $t$ by $n^{*}\left(\lambda_{t}, N_{t}\right)$. The decision maker's choice in period $t$ will determine the number of remaining adopters and the distribution of the public belief in period $t+1$. Hence, we can derive $n^{*}\left(\lambda_{t+1}, N_{t+1}\right)$ accordingly.

Proposition 1 The equilibrium strategy, as a function of the public belief and the number of remaining adopters, is given by the following table, where $n_{e}^{*}$ and $n_{o}^{*}$ denote even and odd integers (Table 1).

In the model, adopters make decisions at most once. When the product is offered to an adopter, his decision does not depend on the number of the remaining adopters. 
Table 1 Equilibrium action in period $t$

\begin{tabular}{lllll}
\hline & $\begin{array}{l}\text { Extremely pessimistic } \\
\left(\lambda_{t}<\underline{\lambda}\right)\end{array}$ & $\begin{array}{l}\text { Pessimistic } \\
\left(\underline{\lambda}<\lambda_{t}<c\right)\end{array}$ & $\begin{array}{l}\text { Optimistic } \\
\left(c<\lambda_{t}<\bar{\lambda}\right)\end{array}$ & $\begin{array}{l}\text { Extremely optimistic } \\
\left(\bar{\lambda}<\lambda_{t}\right)\end{array}$ \\
\hline $\begin{array}{l}\text { Unpromising } \\
\left(\underline{\lambda}<\lambda_{t}<\frac{1}{2}\right)\end{array}$ & 0 & $N_{t}$ & 1 & $N_{t}$ \\
$\begin{array}{c}\text { Promising } \\
\left(\frac{1}{2}<\lambda_{t}<\bar{\lambda}\right)\end{array}$ & 0 & $n_{e}^{*}<N_{t}$ & $n_{o}^{*}<N_{t}$ & $N_{t}$ \\
\hline
\end{tabular}

Hence, in period $t$, the adopters will always reject or take the product if the public belief is below the threshold $\underline{\lambda}$ defined in expression (4) or above the threshold $\bar{\lambda}$ defined in (3), respectively. ${ }^{7}$

If the product is promising, it is likely that its true quality is high. Hence, the decision maker believes an adoption herd will occur almost surely if he makes sufficient many offers in the current period. This also allows the decision maker to minimize the odds of a rejection herd, i.e., minimize the risk that future beliefs are determined by few unlucky draws. The increase in the chance of an adoption herd bears a cost. The more offers the decision maker makes today, the fewer adopters are left. So, the benefit from an adoption herd is smaller. The actual number $n^{*}\left(\lambda_{t}, N_{t}\right)$ depends on this trade-off. As a consequence of the decision maker's strategy, the launch duration of a promising product is at least two periods.

By contrast, if the product is unpromising, allowing adopters to learn from each other will most likely convey bad news about the quality of the product. Hence the decision maker prefers to avoid learning. In particular, if the product is unpromising and adopters are pessimistic, the decision maker's optimal strategy is to suppress any information disclosure and launch the product simultaneously to all adopters. This is because in a sequential launch, only very strong good news from early launches can trigger an adoption herd but mild bad news is damaging enough to trigger a rejection herd. Because the product is unpromising, the latter is more likely to happen. The decision maker therefore will not risk revealing any information, and the product launch lasts only one period.

However, if adopters are optimistic, the decision maker will find it optimal to launch the product to one adopter first and to the remaining ones subsequently. The sequential launch does not aim to favor learning among future adopters (after all it is likely that adopters will learn bad news about the product). Rather, it aims to trigger an adoption herd in the lucky event in which the first adopter happens to receive a good signal. ${ }^{8}$ Given that adopters believe that the product is worth to adopt ex ante, offering the product to only one adopter in period $t$ is not going to trigger a rejection herd but may

\footnotetext{
7 The analysis is the same as in the example with two adopters.

8 In this case, the sequential launch is a way used by the decision maker to favorably manipulate adopters' beliefs.
} 
trigger an adoption herd with a small chance. ${ }^{9}$ The launch duration is at most two periods. Specifically, if the first launch fails, the public belief is updated downward to the range $(\underline{\lambda}, c)$. The decision maker will therefore launch the product simultaneously to the remaining adopters to prevent them from hearing more bad news. Whereas in case of a successful first launch, the product will be offered to and purchased by all remaining adopters.

Corollary 1 When the product is unpromising, an adoption herd may occur during the launch whereas a rejection herd will never happen. Both an adoption herd and a rejection herd may happen during the launch of a promising product.

By proposition 1, when an unpromising product is launched to optimistic adopters, an adoption herd occurs if the first adopter takes the product. The decision maker will not risk revealing too much information and hence will never be trapped in a rejection herd. This result is in sharp difference from Bikhchandani et al. (1992). In Bikhchandani et al. when the sequence is fixed exogenously, either a rejection herd or an adoption herd may occur with a positive probability. In our model, an unpromising product will never be trapped in a rejection herd because of the decision maker's cautious launch strategy.

Contrary to the launch of an unpromising product, when launching a promising product, the decision maker is confident to reveal a lot of information to adopters by making many offers in a single period. Naturally, an adoption herd may happen. But a rejection herd may also occur if the decision maker is unlucky enough to offer the product to many adopters who turn out to receive bad signals. Corollary 1 shows that a promising product may have a higher chance of suffering a complete failure than an unpromising product because of the decision maker's risky launch strategy. ${ }^{10}$

Based on the above description, we can summarize the dynamics. An unpromising product is launched sequentially only if adopters are optimistic. In this case, a successful first launch will trigger an adoption herd. If the first launch fails, the product remains unpromising but adopters become pessimistic. A promising product is always launched sequentially. Furthermore, it is offered to an even number of adopters if they are pessimistic and to an odd number of adopters if they are optimistic. In the former case, either a herd occurs in the next period or the product remains promising and adopters remain pessimistic. This happens when half adopters take the product and half reject it. In the latter case, when failed launches outnumber successful launches

\footnotetext{
9 Suppose, for example, that $\lambda=1 / 4$ and $q=2 / 3$. If the decision maker launches the product to one adopter, the probability of triggering an adoption herd is $5 / 12$; the probability of triggering a rejection herd is zero. If the decision maker launches the product to two adopters, the probability of triggering an adoption herd is 7/36; the probability of triggering a rejection herd is $13 / 36$.

10 Suppose there are three adopters in total, $q=0.7$ and $c=0.65$. Consider a promising product with $\lambda=0.6$ and an unpromising product with $\lambda=0.45$. The decision maker will offer the unpromising product simultaneously to all adopters; he will offer the promising product to two adopters in the first period and to the remaining adopter in the second period if the first two adopters haven't rejected the product. According to the launch strategy, the unpromising product will be rejected by all with probability 0.2 . This happens when all three adopters receive bad signals. In contrast, the promising product will be rejected by all the adopters with probability 0.25 . This happens when the first two adopters receive bad signals. Then, after observing two rejections, the third adopter will also reject the promising product regardless of his own private signal.
} 
by one, the promising product may turn into an unpromising one and adopters will become pessimistic. In summary, if a herd has not occurred in a period, a promising product may turn into an unpromising one but an unpromising product will never become a promising one. From a different perspective, if a herd has not occurred, optimistic adopters may become pessimistic but not vise versa.

\subsection{Discussion}

The role of the precision of signals In our model, the decision maker's ability to influence the learning among adopters is constrained by the precision of signals. Recall that the decision maker can control the information flow from early adopters to later adopters only when people's initial assessment of the product is less extreme, i.e., $\lambda \in(\underline{\lambda}, \bar{\lambda})$. The thresholds for an adoption herd, $\bar{\lambda}$, and a rejection herd, $\underline{\lambda}$, however, are determined by the precision of signals, $q$. Specifically, when signals are more precise, the range $(\underline{\lambda}, \bar{\lambda})$ becomes wider, and hence, the decision maker has more power to influence the remaining adopters' decisions. Intuitively, when signals are very precise, early adopters will put more weight on their private information than on the initial assessment of the product when they make adoption decisions. As a result, the remaining adopters are more likely to learn early adopters' private information from their choices. This gives the decision maker more freedom to influence the remaining adopters' decisions by manipulating the launch sequence.

Heterogenous adopters In the main model, adopters are homogenous ex ante. Hence, an adopter's position in a sequential launch is not important. It is natural to ask what would happen if adopters are heterogenous. In particular, the most interesting extension is to consider how launch strategies are affected by the presence of adopters with different levels of sophistication.

We now analyze the decision maker's launch strategy when some adopters are more sophisticated than others. We modify the simple example discussed in Sect. 4 in the minimal way to give the basic intuition. The decision maker considers launching the product to two potential adopters. Adopter 1 is more sophisticated than adopter 2 . That is, $1 / 2<q_{2}<q_{1}<1$. We henceforth call adopter 1 the expert and adopter 2 the coarse adopter. To simplify the analysis, we set $c=1 / 2$. Given $c=1 / 2$, an expert's adoption decision is in accordance with his signal if the prior belief is in the range $\left(1-q_{1}, q_{1}\right)$. He will always adopt the product if the prior belief is above $q_{1}$ and always reject the product if the prior belief is below $1-q_{1}$. Similarly, a coarse adopter's adoption decision is in accordance with his signal if the prior belief is in the range $\left(1-q_{2}, q_{2}\right)$. Because the signal received by the expert is of a higher precision than that received by the coarse adopter, the expert is less likely to ignore his private signal and herd on the prior belief. As a consequence, the interval $\left(1-q_{2}, q_{2}\right)$ is contained in the interval $\left(1-q_{1}, q_{1}\right)$. (See Fig. 3).

Similar to the example discussed in Sect. 4, the decision maker will offer the product simultaneously to both adopters if the prior belief is above $q_{1}$ and both adopters will always buy. The decision maker will never offer the product to any adopter if the prior belief is below $1-q_{1}$. The decision maker's strategy when the prior belief is not 


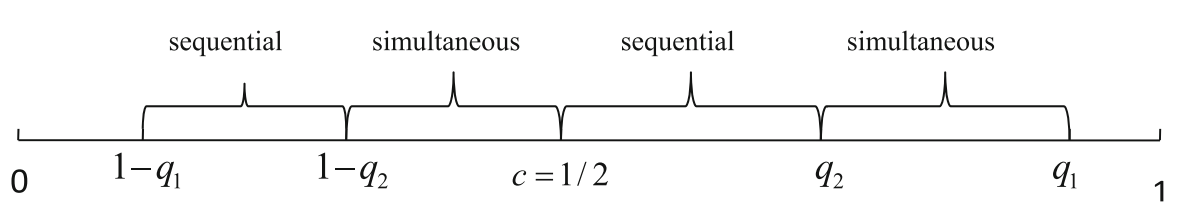

Fig. 3 Heterogenous adopters

extreme is summarized by Fig. 3. We find that the decision maker prefers a sequential offer to a simultaneous offer when the prior belief $\lambda \in\left(1-q_{1}, 1-q_{2}\right) \bigcup\left(c, q_{2}\right)$. Moreover, whenever the decision maker offers the product sequentially, he always offers it first to the expert and then to the coarse adopter. ${ }^{11}$

The intuition for the findings is the following: When the prior belief is in the range of $\left(1-q_{1}, 1-q_{2}\right)$, the coarse adopter will never adopt the product if it is offered simultaneously whereas the expert will adopt the product if and only if he receives a good signal. By contrast, if the decision maker offers the product first to the expert and he adopts it, the decision maker will gain the coarse adopter. This is because the quality of the expert's signal is higher than that of the coarse adopter. Consequently, after hearing the good news from the expert, the coarse adopter will change his mind and adopt the product as well. When the prior belief is in the range of $\left(c, q_{2}\right)$, the decision maker will use the same strategy but he does so for a different reason. As in the main model, when the product is promising, the decision maker will encourage learning because he is confident that the remaining adopter will hear good news from the early one. In this case, the decision maker will offer the product first to the expert because he wants the coarse adopter's decision to be based on the more reliable source of information.

The decision maker will offer the product simultaneously when the prior belief is in the range of either $\left(1-q_{2}, c\right)$ or $\left(q_{2}, q_{1}\right)$. The intuition is the opposite to the intuition for the sequential launch. To see this, consider the case when the prior belief is in $\left(q_{2}, q_{1}\right)$. Given such a prior belief, the coarse adopter will always adopt the product whereas the expert will adopt it if and only if he receives a good signal. Hence, allowing the coarse adopter to learn from the expert, the decision maker will gain nothing but may lose the coarse adopter if he hears bad news from the expert. When the prior belief is in the range of $\left(1-q_{2}, c\right)$, the product is unpromising. Hence, the decision maker will not risk triggering a rejection herd by offering the product sequentially.

\section{Conclusion}

We study a decision maker's product launch strategy when both the decision maker and adopters learn the value of the product through previous adoption decisions. A sequential launch becomes relevant whenever the prior belief about the value of the product is not extreme. In this case, the optimal launch strategy depends on both the adopters' prior beliefs and the adoption costs. Specifically, the decision maker

\footnotetext{
11 The calculation is very similar to that for the simple example and hence is ignored in the text. Detailed calculation will be provided upon request.
} 
will always launch the product sequentially if the prior belief is greater than onehalf (promising product). When the prior belief is smaller than one-half (unpromising product), depending on the adoption cost, the decision maker will either launch the product simultaneously to all adopters or launch it first to one adopter and then to all the remaining adopters.

Our model predicts the following: first, a promising product has a higher chance of suffering a rejection herd than an unpromising product. Second, the launch duration of a promising product is longer than that of an unpromising product. Third, more information about the value of the product will be revealed in the launch of a promising product than that of an unpromising product. Lastly, sequential launch is more likely to occur when adopters' signals are more precise.

Our model provides an explanation for why some well-anticipated products are released in a limited fashion and why sometimes a promising new technology may fail. Moreover, our model can also shed some light on sequential voting procedures. It is well documented that in a primary election, whoever wins the first few states is more likely to generate the momentum to win the nomination. Our model can be applied to a primary election in which a less well-known politician challenges a status quo candidate. Although the sequential order of a primary election is exogenous, our model sheds light on circumstances under which the less well-known politicians benefit from sequential voting.

It would be interesting to extend our work and study how the decision maker's launch strategy is affected by the effect of imperfect information about other agents' previous actions (Celen and Kariv 2005) or about their payoffs (Oyarzun and Ruf 2009), or by the effect of uncertainty about the future state of the word (Moscarini et al. 1998). Another possible extension is to consider the optimal launch strategy when the acquisition of private information among adopters is costly (Burguet and Vives 2000).

\section{Appendix}

We first define some notation. In the appendix, $G_{t} \equiv \lambda_{t} q+\left(1-\lambda_{t}\right)(1-q)$. Similarly, $G_{t+1}^{H} \equiv \lambda^{H}\left(\lambda_{t}\right) q+\left(1-\lambda^{H}\left(\lambda_{t}\right)\right)(1-q)$ and $G_{t+1}^{L} \equiv \lambda^{L}\left(\lambda_{t}\right) q+\left(1-\lambda^{L}\left(\lambda_{t}\right)\right)(1-q)$. The terms $\lambda^{L}\left(\lambda_{t}\right)$ and $\lambda^{H}\left(\lambda_{t}\right)$ are defined by (1) and (2), respectively. Let $\operatorname{Pr}(t \mid M)$ denotes the binomial distribution with parameters $M$, the number of independent experiments, and $q$, the probability of success. Let $\operatorname{Pr}(t \geq T \mid M)$ denote the multinomial distribution. Suppose the decision maker launches the product to $n_{t}$ potential adopters, $n_{t}<N_{t}$. Let $\Delta_{n_{t}}$ denotes the difference between the number of adoptions and the number of rejections among the $n_{t}$ adopters. Since signals are equally precise, having observed the adoption decisions made by adopters in period $t$, the remaining adopters will update their beliefs according to $\Delta_{n_{t}}$.

We explain the belief updating rule, which will be used repeatedly in the proofs. For $c<\lambda_{t}<\bar{\lambda}$, the posterior belief is above $\bar{\lambda}$ if $1 \leq \Delta_{n_{t}}$; it remains $\lambda_{t}$ if $\Delta_{n_{t}}=0$; it is $\lambda^{L}\left(\lambda_{t}\right)$ if $\Delta_{n_{t}}=-1$ and it falls below $\underline{\lambda}$ if $\Delta_{n_{t}} \leq-2$. Similarly, for $\underline{\lambda}<\lambda_{t}<c$, the posterior belief is above $\bar{\lambda}$ if $2 \leq \Delta_{n_{t}}$; it is $\lambda^{H}(\lambda)$ if $\Delta_{n_{t}}=1$; it remains $\lambda_{t}$ if $\Delta_{n_{t}}=0$ and it falls below $\underline{\lambda}$ if $\Delta_{n_{t}} \leq-1$. 
We next present three lemmas, Lemma 1 to Lemma 3, which are used repeatedly in the remaining proofs.

Lemma 1 When the public belief is $\lambda_{t}$, with $\lambda_{t}>\frac{1}{2}$, probability $\operatorname{Pr}\left(\Delta_{2 k-1} \geq 1\right)$ increases in $k$. When $\lambda_{t}<\frac{1}{2}$, probability $\operatorname{Pr}\left(\Delta_{2 k-1} \geq 1\right)$ decreases in $k$. Proof of lemma 1

$$
\begin{aligned}
\operatorname{Pr}\left(\Delta_{2 k-1} \geq 1\right) & =\sum_{i=k}^{2 k-1}\left(\lambda_{t} q^{i}(1-q)^{2 k-1-i}+\left(1-\lambda_{t}\right) q^{2 k-1-i}(1-q)^{i}\right) C_{i}^{2 k-1} \\
& =\left(1-\lambda_{t}\right)+\operatorname{Pr}(t \geq k \mid 2 k-1)\left(2 \lambda_{t}-1\right) \\
\operatorname{Pr}\left(\Delta_{2 k+1} \geq 1\right)-\operatorname{Pr}\left(\Delta_{2 k-1} \geq 1\right) & =\left(2 \lambda_{t}-1\right) q^{k}(1-q)^{k}(2 q-1) C_{k}^{2 k-1} .
\end{aligned}
$$

Since $q \in\left(\frac{1}{2}, 1\right)$, the difference $\operatorname{Pr}\left(\Delta_{2 k+1} \geq 1\right)-\operatorname{Pr}\left(\Delta_{2 k-1} \geq 1\right)$ is positive when $\lambda_{t}>\frac{1}{2}$ and negative when $\lambda_{t}<\frac{1}{2}$.

Lemma 2 When the public belief is $\lambda_{t}$, with $\lambda_{t}>\frac{1}{2}$, probability $\operatorname{Pr}\left(\Delta_{2 k} \geq 2\right)$ increases in $k$.

Proof of Lemma 2 The probability $\operatorname{Pr}\left(\Delta_{2 k} \geq 2\right)$ can be written as

$$
\left(1-\lambda_{t}\right)+\left(2 \lambda_{t}-1\right) \operatorname{Pr}(t \geq k+1 \mid 2 k)-\left(1-\lambda_{t}\right) \operatorname{Pr}(t=k \mid 2 k) .
$$

So, the difference $\operatorname{Pr}\left(\Delta_{2 k+2} \geq 2\right)-\operatorname{Pr}\left(\Delta_{2 k} \geq 2\right)$ is

$$
\left(2 \lambda_{t}-1\right) \operatorname{Pr}(t=k \mid 2 k) q\left(q-\frac{k(1-q)}{k+1}\right)+\left(1-\lambda_{t}\right) \operatorname{Pr}(t=k \mid 2 k)\left(1-\frac{2 q(1-q)(2 k+1)}{k+1}\right) .
$$

Since $\left(\frac{2 q(1-q)(2 k+1)}{k+1}\right)<4 q(1-q)<1,\left(1-\lambda_{t}\right) \operatorname{Pr}(t=k \mid 2 k)\left(1-\frac{2 q(1-q)(2 k+1)}{k+1}\right)>0$. When $\lambda_{t}>\frac{1}{2},\left(2 \lambda_{t}-1\right) \operatorname{Pr}(t=k \mid 2 k) q\left(q-\frac{(1-q) k}{k+1}\right)>0$. Hence $\operatorname{Pr}\left(\Delta_{2 k+2} \geq 2\right)>$ $\operatorname{Pr}\left(\Delta_{2 k} \geq 2\right)$.

Lemma 3 When the public belief is $\lambda_{t}$, with $\lambda_{t}<\frac{1}{2}$, the term $\operatorname{Pr}\left(\Delta_{2 k} \geq 2\right)+$ $\operatorname{Pr}\left(\Delta_{2 k}=0\right) G_{t}$ decreases in $k$.

Proof of Lemma 3 Let $J(k) \equiv \operatorname{Pr}\left(\Delta_{2 k} \geq 2\right)+\operatorname{Pr}\left(\Delta_{2 k}=0\right) G_{t}$. The difference

$$
\begin{aligned}
& J(k+1)-J(k)=\operatorname{Pr}\left(\Delta_{2 k+2} \geq 2\right) \\
& \quad-\operatorname{Pr}\left(\Delta_{2 k} \geq 2\right)-\left(\operatorname{Pr}\left(\Delta_{2 k}=0\right)-\operatorname{Pr}\left(\Delta_{2 k+2}=0\right)\right) G_{t} .
\end{aligned}
$$

Substitute $\operatorname{Pr}\left(\Delta_{2 k+2} \geq 2\right)-\operatorname{Pr}\left(\Delta_{2 k} \geq 2\right)\left(\right.$ See Lemma 2) and $\operatorname{Pr}\left(\Delta_{2 k}=0\right)-$ $\operatorname{Pr}\left(\Delta_{2 k+2}=0\right)$. The difference

$$
J(k+1)-J(k)=\frac{q(1-q)(2 q(2 k+1)-1)\left(2 \lambda_{t}-1\right) \operatorname{Pr}(t=k \mid 2 k, q)}{k+1} .
$$

Since $\frac{q(1-q)(2 q(2 k+1)-1)}{k+1}>0, J(k+1)-J(k)$ is negative. 
Proof of Proposition 1 The proof is divided into two cases: optimal launch strategy for an unpromising product and that for a promising product.

Case 1: unpromising product. The proof is divided into two steps. Step 1 shows $n^{*}\left(\lambda_{t}, N_{t}\right)=N_{t}$ if $\lambda_{t}<c$. Step 2 shows $n^{*}\left(\lambda_{t}, N_{t}\right)=1$ if $\lambda_{t}>c$.

Step 1. We first show it is never optimal to start a sequential launch with an odd number of adopters. Suppose the decision maker offers the product to $2 k+1$ adopters in period $t$, where $k$ is a natural number and $2 k+1<N_{t}$. His expected payoff $\pi\left(n_{t}=2 k+1\right)$ is

$$
(2 k+1) G_{t}+\operatorname{Pr}\left(\Delta_{2 k+1} \geq 3\right)\left(N_{t}-2 k-1\right)+\operatorname{Pr}\left(\Delta_{2 k+1}=1\right) V\left(\lambda_{t+1}^{H}, N_{t}-2 k-1\right) .
$$

Since $V\left(\lambda^{H}\left(\lambda_{t}\right), N_{t}-2 k-1\right)<\left(N_{t}-2 k-1\right)$,

$$
\pi\left(n_{t}=2 k+1\right)<(2 k+1) G_{t}+\operatorname{Pr}\left(\Delta_{2 k+1} \geq 1\right)\left(N_{t}-2 k-1\right) .
$$

By lemma 1 , we know $\operatorname{Pr}\left(\Delta_{2 k+1} \geq 1\right)$ decreases in $k$ when $\lambda<1 / 2$. Hence, $\pi\left(n_{t}=\right.$ $2 k+1)<(2 k+1) G_{t}+G_{t}\left(N_{t}-2 k-1\right)=N_{t} G_{t}=\pi\left(n_{t}=N_{t}\right)$.

Next, we show it is never optimal to start a sequential launch with an even number of adopters. We show this by contradiction. Suppose the decision maker starts a sequential launch with an even number of adopters, and he launches the product to $n_{T}$ adopters in period $T$, the last period, with $T>2$, and $n_{T-1}$ in period $T-1$. Let $\pi_{T-1}$ denotes the decision maker's payoff in period $T-1$ from this strategy. We show that the decision maker's payoff from launching the product to $n_{T}+n_{T-1}$ adopters in period $T-1$ is greater than $\pi_{T-1}$, where $\pi_{T-1}$ is

$$
n_{T-1} G_{t}+\operatorname{Pr}\left(\Delta_{n_{T-1}} \geq 2\right) n_{T}+\operatorname{Pr}\left(\Delta_{n_{T-1}}=1\right) n_{T} G_{t+1}^{H}+\operatorname{Pr}\left(\Delta_{n_{T-1}}=0\right) n_{T} G_{t} .
$$

We have shown it is never optimal for the seller to launch the product to an odd number of adopters. Therefore, the launch can last to period $T-1$ only if the seller keeps launching the product to an even number of adopters and half adopters reject the product while the other half accept the product in each previous period. Otherwise, a herd will occur before period $T-1$. By this argument, the prior belief in period $T-1$ remains $\lambda_{t}$. By the argument in the last paragraph, $n_{T-1}$ must be an even number and hence, $\operatorname{Pr}\left(\Delta_{n_{T-1}}=1\right)=0$. As a consequence, $\pi_{T-1}=n_{T-1} G_{t}+\left(\operatorname{Pr}\left(\Delta_{n_{T-1}} \geq\right.\right.$ $\left.2)+\operatorname{Pr}\left(\Delta_{n_{T-1}}=0\right) G_{t}\right) n_{T}$. By Lemma 3 , the term $\operatorname{Pr}\left(\Delta_{n_{T-1}} \geq 2\right)+\operatorname{Pr}\left(\Delta_{n_{T-1}}=0\right) G_{t}$ decreases in $n_{T-1}$. Hence, an upper bound of $\operatorname{Pr}\left(\Delta_{n_{T-1}} \geq 2\right)+\operatorname{Pr}\left(\Delta_{n_{T-1}}=0\right) G_{t}$ is $\operatorname{Pr}\left(\Delta_{2} \geq 2\right)+\operatorname{Pr}\left(\Delta_{2}=0\right) G_{t}=G_{t}-q(1-q)\left(1-2 G_{t}\right)$. When $\lambda<1 / 2, G_{t}<1 / 2$. Hence, $\operatorname{Pr}\left(\Delta_{2} \geq 2\right)+\operatorname{Pr}\left(\Delta_{2}=0\right) G_{t}<G_{t}$. As a consequence, the payoff $\pi_{T-1}<$ $n_{T-1} G_{t}+n_{T} G_{t}=\left(n_{T}+n_{T-1}\right) G_{t}$.

Step 2. We show this step by induction. We first show if $N_{t}=2, n^{*}\left(\lambda_{t}, 2\right)=1$. The payoff from a simultaneous launch is $\pi\left(n_{t}=2\right)=2 G_{t}$. The payoff from a sequential launch is $\pi\left(n_{t}=1\right)=2 G_{t}+\left(1-G_{t}\right) G_{t+1}^{L}$. The difference $\pi\left(n_{t}=1\right)-\pi\left(n_{t}=\right.$ 2) $=q(1-q)>0$.

Next, we show given $n^{*}\left(\lambda_{t}, N_{t}-1\right)=1$, for $N_{t}-1>2, n^{*}\left(\lambda_{t}, N_{t}\right)=1$. We first argue $n\left(\lambda_{t}, N_{t}\right)=1$ is more profitable than $n\left(\lambda_{t}, N_{t}\right)=2 n+1$ for $n=1,2, . . n$ max , with $2 n+1 \leq N_{t}$. The payoff $\pi\left(n_{t}=1\right)=G_{t} N_{t}+\left(1-G_{t}\right) V\left(\lambda^{L}\left(\lambda_{t}\right), N_{t}-1\right)$. When 
$c<\lambda_{t}<\min \left(\bar{\lambda}, \frac{1}{2}\right)$, the posterior belief after receiving a bad signal is $\lambda^{L}\left(\lambda_{t}\right)$, where $\underline{\lambda}<\lambda^{L}\left(\lambda_{t}\right)<\min \left(c, \frac{1}{2}\right)$. According to step $1, V\left(\lambda^{L}\left(\lambda_{t}\right), N_{t}-1\right)=\left(N_{t}-1\right) G_{t+1}^{L}$. Consequently, $\pi\left(n_{t}=1\right)$ is $G_{t} N_{t}+q(1-q)\left(N_{t}-1\right)$. The payoff $\pi\left(n_{t}=2 n+1\right)$ is

$(2 n+1) G_{t}+\operatorname{Prob}\left(\Delta_{2 n+1} \geq 1\right)\left(N_{t}-2 n-1\right)+\operatorname{Prob}\left(\Delta_{2 n+1}=-1\right)\left(N_{t}-2 n-1\right) G_{t+1}^{L}$.

The difference $\pi\left(n_{t}=1\right)-\pi\left(n_{t}=2 n+1\right)$ is

$$
\left(N_{t}-2 n-1\right)\left(G_{t}-\operatorname{Pr}\left(\Delta_{2 n+1} \geq 1\right)-\operatorname{Pr}\left(\Delta_{2 n+1}=-1\right) G_{t+1}^{L}\right)+q(1-q)\left(N_{t}-1\right) .
$$

Substitute $\operatorname{Prob}\left(\Delta_{2 n+1} \geq 1\right)=\left(1-\lambda_{t}\right)+\operatorname{Pr}(t \geq n+1 \mid 2 n+1, q)\left(2 \lambda_{t}-1\right)$ and $\operatorname{Pr}\left(\Delta_{2 n+1}=-1\right)=C_{n}^{2 n+1} q^{n}(1-q)^{n}\left(1-G_{t}\right)$ into $\pi\left(n_{t}=1\right)-\pi\left(n_{t}=2 n+1\right)$. The difference becomes

$$
\begin{aligned}
& \pi\left(n_{t}=1\right)-\pi\left(n_{t}=2 n+1\right) \\
& \quad=\left(N_{t}-2 n-1\right)\left(\left(1-2 \lambda_{t}\right)(\operatorname{Pr}(t \geq n+1 \mid 2 n+1)-q)-C_{n}^{2 n+1} q^{n+1}(1-q)^{n+1}\right) \\
& \quad+q(1-q)\left(N_{t}-1\right) .
\end{aligned}
$$

Given $\lambda_{t} \in\left(0, \frac{1}{2}\right)$ and $q \in(0.5,1),\left(1-2 \lambda_{t}\right)(\operatorname{Pr}(t \geq n+1 \mid 2 n+1)-q)>0$. Hence

$$
\begin{aligned}
& \pi\left(n_{t}=1\right)-\pi\left(n_{t}=2 n+1\right) \\
& \quad>q(1-q)\left(N_{t}-1\right)-C_{n}^{2 n+1} q^{n+1}(1-q)^{n+1}\left(N_{t}-2 n-1\right) \\
& \quad=q(1-q)\left(N_{t}-2 n-1\right)\left(\frac{N_{t}-1}{N_{t}-1-2 n}-C_{n}^{2 n+1} q^{n}(1-q)^{n}\right) .
\end{aligned}
$$

The term $C_{n}^{2 n+1} q^{n}(1-q)^{n}$ is equal to $\frac{2 n+1}{n+1} \operatorname{Pr}(t=n \mid 2 n, q)$ which is less than $2 \operatorname{Pr}(t=$ $n \mid 2 n, q)$. Since $\operatorname{Pr}(t=n \mid 2 n, q)$ decreases in $n, \operatorname{Pr}(t=n \mid 2 n, q) \leq 2 q(1-q)$. Accordingly, $C_{n}^{2 n+1} q^{n}(1-q)^{n}<4 q(1-q)<1$. Because $\frac{N_{t}-1}{N_{t}-1-2 n}>1, \pi\left(n_{t}=1\right)-\pi\left(n_{t}=\right.$ $2 n+1)>0$.

Now, we show $n_{t}=1$ is more profitable than $n_{t}=2 n$, for $n=1,2, \ldots, n$ max, with $2 n \leq N_{t}$. The payoff $\pi\left(n_{t}=2 n\right)$ is

$\pi\left(n_{t}=2 n\right)=2 n G_{t}+\operatorname{Prob}\left(\Delta_{2 n} \geq 2\right)\left(N_{t}-2 n\right)+\operatorname{Prob}\left(\Delta_{2 n}=0\right) V\left(\lambda_{t}, N_{t}-2 n\right)$.

The difference $\pi\left(n_{t}=1\right)-\pi\left(n_{t}=2 n\right)$ is

$\left(N_{t}-2 n\right)\left(G_{t}-\operatorname{Pr}\left(\Delta_{2 n} \geq 2\right)\right)+q(1-q)\left(N_{t}-1\right)-\operatorname{Pr}\left(\Delta_{2 n}=0\right) V\left(\lambda_{t}, N_{t}-2 n\right)$.

By the hypothesis $n^{*}\left(\lambda_{t}, N_{t}-1\right)=1$,

$$
\begin{aligned}
& V\left(\lambda_{t}, N_{t}-2 n\right) \\
& =G_{t}\left(N_{t}-2 n\right)+\left(1-G_{t}\right) G_{t+1}^{L}\left(N_{t}-2 n\right)=G_{t}\left(N_{t}-2 n\right)+q(1-q)\left(N_{t}-2 n\right) .
\end{aligned}
$$


Substitute $V\left(\lambda_{t}, N_{t}-2 n\right)$ into $\pi\left(n_{t}=1\right)-\pi\left(n_{t}=2 n\right)$, the difference boils down to

$$
\begin{aligned}
& \pi\left(n_{t}=1\right)-\pi\left(n_{t}=2 n\right) \\
& =\left(N_{t}-2 n\right)\left(G_{t}\left(1-\operatorname{Pr}\left(\Delta_{2 n}=0\right)\right)-\operatorname{Pr}\left(\Delta_{2 n} \geq 2\right)\right) \\
& \quad+q(1-q)\left(N_{t}-2 n\right)\left(\frac{N_{t}-1}{N_{t}-2 n}-\operatorname{Pr}(t=n \mid 2 n, q)\right) .
\end{aligned}
$$

We have shown in step 1 that $G_{t}\left(1-\operatorname{Pr}\left(\Delta_{2 n}=0\right)\right)-\operatorname{Pr}\left(\Delta_{2 n} \geq 2\right)>0$. The ratio $\frac{N_{t}-1}{N_{t}-2 n}>1$ for $n=1,2, \ldots, n$ max. The probability $\operatorname{Pr}(t=n \mid 2 n, q)$ decreases in $n$, which implies $\operatorname{Pr}(t=n \mid 2 n, q) \leq 2 q(1-q)<1$. Therefore, $\frac{N_{t}-1}{N_{t}-2 n}-\operatorname{Pr}(t=$ $n \mid 2 n, q)>0$. Consequently, $\pi\left(n_{t}=1\right)-\pi\left(n_{t}=2 n\right)>0$.

Case 2: promising product. The proof is divided into two steps. Step 1 shows when $\lambda_{t}<c$, the decision maker will start a sequential launch with an even number of adopters. Step 2 shows when $c<\lambda_{t}$, the decision maker will start a sequential launch with an odd number of adopters.

Step 1. We first show launching the product first to an even number of adopters $2 k$, where $k$ is a natural number and $2 k \leq N_{t}$, is more profitable than to an odd number of adopters $2 k+1$. The decision maker's expected payoff from launching the product to $2 k$ adopters is

$$
\pi\left(n_{t}=2 k\right)=2 k G_{t}+\operatorname{Pr}\left(\Delta_{2 k} \geq 2\right)\left(N_{t}-2 k\right)+\operatorname{Pr}\left(\Delta_{2 k}=0\right) V\left(\lambda_{t}, N_{t}-2 k\right) .
$$

His payoff from launching the product to $2 k+1,2 k+1 \leq N_{t}$, is

$$
\begin{aligned}
& \pi\left(n_{t}=2 k+1\right) \\
& =(2 k+1) G_{t}+\operatorname{Pr}\left(\Delta_{2 k+1} \geq 3\right)\left(N_{t}-2 k-1\right) \\
& \quad+\operatorname{Pr}\left(\Delta_{2 k+1}=1\right) V\left(\lambda^{H}\left(\lambda_{t}\right), N_{t}-2 k-1\right) .
\end{aligned}
$$

The term $\frac{\pi\left(n_{t}=2 k\right)-\pi\left(n_{t}=2 k+1\right)}{\operatorname{Pr}\left(\Delta_{2 k}=0\right)}$ can be written as

$$
\begin{aligned}
& \frac{\pi\left(n_{t}=2 k\right)-\pi\left(n_{t}=2 k+1\right)}{\operatorname{Pr}\left(\Delta_{2 k}=0\right)} \\
& =\frac{\operatorname{Pr}\left(\Delta_{2 k} \geq 2\right)-G_{t}}{\operatorname{Pr}\left(\Delta_{2 k}=0\right)}+\frac{\operatorname{Pr}\left(\Delta_{2 k} \geq 2\right)-\operatorname{Pr}\left(\Delta_{2 k+1} \geq 3\right)}{\operatorname{Pr}\left(\Delta_{2 k}=0\right)}\left(N_{t}-2 k-1\right) \\
& \quad+V\left(\lambda_{t}, N_{t}-2 k\right)-\frac{\operatorname{Pr}\left(\Delta_{2 k+1}=1\right)}{\operatorname{Pr}\left(\Delta_{2 k}=0\right)} V\left(\lambda^{H}\left(\lambda_{t}\right), N_{t}-2 k-1\right) .
\end{aligned}
$$

Substitute $\operatorname{Pr}\left(\Delta_{2 k} \geq 2\right), \operatorname{Pr}\left(\Delta_{2 k+1} \geq 3\right), \operatorname{Pr}\left(\Delta_{2 k+1}=1\right)$ and $\operatorname{Pr}\left(\Delta_{2 k}=0\right)$ into $\frac{\pi\left(n_{t}=2 k\right)-\pi\left(n_{t}=2 k+1\right)}{\operatorname{Pr}\left(\Delta_{2 k}=0\right)}$. It becomes 


$$
\begin{aligned}
\frac{\pi\left(n_{t}=2 k\right)-\pi\left(n_{t}=2 k+1\right)}{\operatorname{Pr}\left(\Delta_{2 k}=0\right)} \\
=\frac{\operatorname{Pr}\left(\Delta_{2 k} \geq 2\right)-G_{t}}{\operatorname{Pr}\left(\Delta_{2 k}=0\right)}+\frac{k G_{t}}{k+1}\left(N_{t}-2 k-1\right)+V\left(\lambda_{t}, N_{t}-2 k\right) \\
\quad-\frac{(2 k+1) G_{t}}{k+1} V\left(\lambda^{H}\left(\lambda_{t}\right), N_{t}-2 k-1\right) .
\end{aligned}
$$

Given $\lambda_{t}$ and $N_{t}$, the decision maker can choose to launch the product to one adopter in period $t$ and follow the equilibrium launch strategy in period $t+1$. Hence, his continuation payoff $V\left(\lambda_{t}, N_{t}-2 k\right)>G_{t}+G_{t} V\left(\lambda^{H}\left(\lambda_{t}\right), N_{t}-2 k-1\right)$. Because $\lambda_{t} \in\left(\frac{1}{2}, 1\right)$ and $q \in\left(\frac{1}{2}, 1\right)$, when the public belief is $\lambda^{H}\left(\lambda_{t}\right)$ and the number of the remaining adopters is $N_{t}-2 k-1$, the decision maker's continuation payoff $V\left(\lambda^{H}\left(\lambda_{t}\right)\right.$, $\left.N_{t}-2 k-1\right)$ is smaller than $\left(N_{t}-2 k-1\right)$. Accordingly, $\frac{\pi\left(n_{t}=2 k\right)-\pi\left(n_{t}=2 k+1\right)}{\operatorname{Pr}\left(\Delta_{2 k}=0\right)}>$ $\frac{\operatorname{Pr}\left(\Delta_{2 k} \geq 2\right)-G_{t}}{\operatorname{Pr}\left(\Delta_{2 k}=0\right)}+G_{t}$. By Lemma 2, when $\lambda_{t}>\frac{1}{2}, \operatorname{Pr}\left(\Delta_{2 k} \geq 2\right)$ increases in $k$. It is easy to show $\operatorname{Pr}\left(\Delta_{2 k}=0\right)$ decreases in $k$. Hence $\frac{\operatorname{Pr}\left(\Delta_{2 k} \geq 2\right)-G_{t}}{\operatorname{Pr}\left(\Delta_{2 k}=0\right)}+G_{t}$ increases in $k$. When $k=1$,

$$
\frac{\operatorname{Pr}\left(\Delta_{2} \geq 2\right)-G_{t}}{\operatorname{Pr}\left(\Delta_{2}=0\right)}+G_{t}=1 / 2(2 q-1)\left(2 \lambda_{t}-1\right)>0 .
$$

Therefore, $\pi\left(n_{t}=2 k\right)>\pi\left(n_{t}=2 k+1\right)$.

Next, we show it is never optimal to launch the product first to a single adopter. We prove this by showing $\pi\left(n_{t}=1\right)<\pi\left(n_{t}=2\right)$. The payoff $\pi\left(n_{t}=1\right)=$ $G_{t}+G_{t} V\left(\lambda^{H}\left(\lambda_{t}\right), N_{t}-1\right)$ and $\pi\left(n_{t}=2\right)=2 G_{t}+\left(\lambda_{t} q^{2}+\left(1-\lambda_{t}\right)(1-q)^{2}\right)$ $\left(N_{t}-2\right)+2 q(1-q) V\left(\lambda_{t}, N_{t}-2\right)$. The difference

$$
\begin{aligned}
& \pi\left(n_{t}=2\right)-\pi\left(n_{t}=1\right) \\
& =G_{t}+\left(G_{t}-q(1-q)\right)\left(N_{t}-2\right)+2 q(1-q) V\left(\lambda_{t}, N_{t}-2\right) \\
& \quad-G_{t} V\left(\lambda^{H}\left(\lambda_{t}\right), N_{t}-1\right) .
\end{aligned}
$$

The continuation payoff $v\left(\lambda_{t}, N_{t}-2\right)$ is at least the payoff from a simultaneous launch to $N_{t}-2$ adopters, which is $\left(N_{t}-2\right) G_{t}$. Hence, $\pi\left(n_{t}=2\right)-\pi\left(n_{t}=1\right)$ is at least $\left(N_{t}-2\right)\left(2 G_{t}-1\right) q(1-q)$. Given $\frac{1}{2}<\lambda_{t}, G_{t}>\frac{1}{2}$. Consequently, $\pi\left(n_{t}=2\right)>$ $\pi\left(n_{t}=1\right)$.

Lastly, we show it is never optimal to launch the product simultaneously. The decision maker's expected payoff from a simultaneous launch is $\pi\left(n_{t}=N_{t}\right)=$ $G_{t} N_{t}$; his payoff from a sequential launch started with two adopters $\pi\left(n_{t}=2\right)$ is described in the above paragraph. Since $v\left(\lambda_{t}, N_{t}-2\right)$ is at least $G_{t}\left(N_{t}-2\right)$, the payoff from a simultaneous launch, the difference $\pi\left(n_{t}=2\right)-\pi\left(n_{t}=N_{t}\right)$ is at least $q(1-q)(2 q-1)\left(2 \lambda_{t}-1\right)$. Because $\frac{1}{2}<q$ and $\frac{1}{2}<\lambda_{t}, \pi\left(n_{t}=2\right)>\pi\left(n_{t}=N_{t}\right)$.

Step 2 . We show it is more profitable to launch the product first to an odd number of adopters than to an even number of adopters. The decision maker's expected payoff 
from offering the product to an odd number of adopters $2 k-1,2 k-1 \leq N_{t}$, is

$$
\begin{aligned}
& \pi\left(n_{t}=2 k-1\right) \\
& =(2 k-1) G_{t}+\operatorname{Pr}\left(\Delta_{2 k-1} \geq 1\right)\left(N_{t}-2 k+1\right) \\
& \quad+\operatorname{Pr}\left(\Delta_{2 k-1}=-1\right) V\left(\lambda^{L}\left(\lambda_{t}\right), N_{t}-2 k+1\right) .
\end{aligned}
$$

His payoff from launching the product to an even number of adopters $2 k, 2 k<N_{t}$, is

$$
\pi\left(n_{t}=2 k\right)=2 k G_{t}+\operatorname{Pr}\left(\Delta_{2 k} \geq 2\right)\left(N_{t}-2 k\right)+\operatorname{Pr}\left(\Delta_{2 k}=0\right) V\left(\lambda_{t}, N_{t}-2 k\right) .
$$

Substitute $\pi\left(n_{t}=2 k-1\right)$ and $\pi\left(n_{t}=2 k\right)$,

$$
\begin{aligned}
& \frac{\pi\left(n_{t}=2 k-1\right)-\pi\left(n_{t}=2 k\right)}{\operatorname{Pr}\left(\Delta_{2 k-1}=-1\right)} \\
& =\frac{\operatorname{Pr}\left(\Delta_{2 k-1} \geq 1\right)-G_{t}}{\operatorname{Pr}\left(\Delta_{2 k-1}=-1\right)}+\frac{\operatorname{Pr}\left(\Delta_{2 k-1} \geq 1\right)-\operatorname{Pr}\left(\Delta_{2 k} \geq 2\right)}{\operatorname{Pr}\left(\Delta_{2 k-1}=-1\right)}\left(N_{t}-2 k\right) \\
& \quad+V\left(\lambda^{L}\left(\lambda_{t}\right), N_{t}-2 k+1\right)-\frac{\operatorname{Pr}\left(\Delta_{2 k}=0\right)}{\operatorname{Pr}\left(\Delta_{2 k-1}=-1\right)} V\left(\lambda_{t}, N_{t}-2 k\right) .
\end{aligned}
$$

Substitute $\operatorname{Pr}\left(\Delta_{2 k}=0\right), \operatorname{Pr}\left(\Delta_{2 k-1}=-1\right), \operatorname{Pr}\left(\Delta_{2 k} \geq 2\right)$ and $\operatorname{Pr}\left(\Delta_{2 k-1} \geq 1\right)$ into $\frac{\pi\left(n_{t}=2 k-1\right)-\pi\left(n_{t}=2 k\right)}{\operatorname{Pr}\left(\Delta_{2 k-1}=-1\right)}$. It becomes

$$
\begin{aligned}
& \frac{\pi\left(n_{t}=2 k-1\right)-\pi\left(n_{t}=2 k\right)}{\operatorname{Pr}\left(\Delta_{2 k-1}=-1\right)} \\
& =\frac{\operatorname{Pr}\left(\Delta_{2 k-1} \geq 1\right)-G_{t}}{\operatorname{Pr}\left(\Delta_{2 k-1}=-1\right)}+\frac{q(1-q)}{1-G_{t}}\left(N_{t}-2 k\right)+V\left(\lambda^{L}\left(\lambda_{t}\right), N_{t}-2 k+1\right) \\
& \quad-\frac{2 q(1-q)}{1-G_{t}} V\left(\lambda_{t}, N_{t}-2 k\right) .
\end{aligned}
$$

When the public belief is $\lambda^{L}\left(\lambda_{t}\right)$ and the number of the remaining adopters is $N_{t}-$ $2 k+1$, the decision maker can choose to launch the product to one adopter in the current period and follow the optimal strategy in the next period. Hence, $V\left(\lambda^{L}\left(\lambda_{t}\right), N_{t}-\right.$ $2 k+1) \geq G_{t+1}^{L}+G_{t+1}^{L} V\left(\lambda_{t}, N_{t}-2 k\right)$. Given $\lambda_{t} \in(0,1)$ and $q \in\left(\frac{1}{2}, 1\right)$, the decision maker's continuation payoff $V\left(\lambda_{t}, N_{t}-2 k\right)$ is less than $\left(N_{t}-2 k\right)$. As a result

$$
\begin{aligned}
& \frac{\pi\left(n_{t}=2 k-1\right)-\pi\left(n_{t}=2 k\right)}{\operatorname{Pr}\left(\Delta_{2 k-1}=-1\right)} \\
& >\frac{\operatorname{Pr}\left(\Delta_{2 k-1} \geq 1\right)-G_{t}}{\operatorname{Pr}\left(\Delta_{2 k-1}=-1\right)}+G_{t+1}^{L}+\left(G_{t+1}^{L}-\frac{q(1-q)}{1-G_{t}}\right) V\left(\lambda_{t}, N_{t}-2 k\right) \\
& =\frac{\operatorname{Pr}\left(\Delta_{2 k-1} \geq 1\right)-G_{t}}{\operatorname{Pr}\left(\Delta_{2 k-1}=-1\right)}+G_{t+1}^{L} .
\end{aligned}
$$

By Lemma $1, \operatorname{Pr}\left(\Delta_{2 k-1} \geq 1\right) \geq \operatorname{Pr}\left(\Delta_{1} \geq 1\right)$. Hence, $\operatorname{Pr}\left(\Delta_{2 k-1} \geq 1\right)-G_{t} \geq 0$. Accordingly $\pi\left(n_{t}=2 k-1\right)>\pi\left(n_{t}=2 k\right)$. 
Next, we show it is never optimal to launch the product simultaneously. The decision maker's payoff from offering the product simultaneously is $\pi\left(n_{t}=N_{t}\right)=G_{t} N_{t}$. His payoff from offering the product first to one adopter is $\pi\left(n_{t}=1\right)=G_{t} N_{t}+(1-$ $\left.G_{t}\right) V\left(\lambda^{L}\left(\lambda_{t}\right), N_{t}-1\right)$. Clearly, as $0<V\left(\lambda^{L}\left(\lambda_{t}\right), N_{t}-1\right), \pi\left(n_{t}=N_{t}\right)<\pi\left(n_{t}=1\right)$.

\section{References}

Bala, V., Goyal, S.: Conformism and diversity under social learning. Econ Theory 17, 101-120 (2001)

Banerjee, V.A.: A simple model of herd behavior. Q J Econ 107, 797-817 (1992)

Bar-Isaac, H.: Reputation and survival: learning in a dynamic signalling model. Rev Econ Stud 70, 231251 (2003)

Bergemann, D., Valimaki, J.: Learning and strategic pricing. Econometrica 64, 1125-1149 (1996)

Bergemann, D., Valimaki, J.: Market diffusion with two-sided learning. RAND J Econ 28, 773-795 (1997)

Bergemann, D., Valimaki, J.: Experimentation in markets. Rev Econ Stud 67, 213-234 (2000)

Bhalla, M.: Waterfall versus Sprinkler Product Launch Strategy: Influencing the Herd. Working paper (2008)

Bikhchandani, S., Hirshleifer, D., Welch, I.: A theory of fads, fashion, custom, and cultural change as informational cascades. J Polit Econ 100, 992-1026 (1992)

Bose, S., Orosel, G., Ottaviani, M., Vesterlund, L.: Dynamic monopoly pricing and herding. RAND J Econ 37, 910-928 (2006)

Bose, S., Orosel, G., Ottaviani, M., Vesterlund, L.: Monopoly pricing in the binary herding model. Econ Theory 37, 203-241 (2008)

Burguet, R., Vives, X.: Social learning and costly information acquisition. Econ Theory 15, 185-205 (2000)

Celen, B., Kariv, S.: An experimental test of observational learning under imperfect information. Econ Theory 26, 677-699 (2005)

Coopland, A.: Learning dynamics with private and public Signals. Econ Theory 31, 523-538 (2007)

Gill, D., Sgroi, D.: Sequential decisions with tests. Games Econ Behav 36, 663-678 (2008)

Gill, D., Sgroi, D.: The Optimal Choice of Pre-launch Reviewer: How Best to Transmit Information using Tests and Conditional Pricing. Working paper (2008)

Moscarini, G., Ottaviani, M., Smith, L.: Social learning in a changing world. Econ Theory 11, 657665 (1998)

Ottaviani, M., Prat, A.: The value of public information in monopoly. Econometrica 69, 1673-1683 (2001)

Oyarzun, C., Ruf, J.: Monotone imitation. Econ Theory 41, 411-441 (2009)

Sgroi, D.: Optimizing information in the herd: guinea pigs, profits, and welfare. Games Econ Behav 39, 137166 (2002)

Taylor, C.: Time-on-the-market as a sign of quality. Rev Econ Stud 66, 555-578 (1999) 